\title{
GiraffPlus: Combining social interaction and long term monitoring for promoting independent living
}

\author{
S. Coradeschi ${ }^{1}$, A. Cesta ${ }^{2}$, G. Cortellessa ${ }^{2}$, L. Coraci ${ }^{2}$, J. Gonzalez ${ }^{3}$, L. Karlsson ${ }^{1}$, F. Furfari ${ }^{4}$, \\ A. Loutfi ${ }^{1}$, A. Orlandini ${ }^{2}$, F. Palumbo ${ }^{4}$, F. Pecora ${ }^{1}$, S. von Rump ${ }^{5}$, A. Štimec ${ }^{7}$, J. Ullberg ${ }^{1}$, B. Ötslund ${ }^{6}$, \\ ${ }^{1}$ Örebro University, AASS, Sweden, ${ }^{2}$ CNR-ISTC, Rome, Italy, ${ }^{3}$ Malaga University, Spain, ${ }^{4}$ CNR-ISTI, \\ Pisa, Italy, ${ }^{5}$ Giraff AB, Sweden, ${ }^{6}$ Lund University, Sweden, XRPOISD, Slovenia \\ silvia.coradeschi@oru.se, amedeo.cesta@istc.cnr.it, gabriella.cortellessa@istc.cnr.it, \\ luca.coraci@istc.cnr.it,jgonzalez@ctima.uma.es, lars.karlsson@oru.se, francesco.furfari@isti.cnr.it, \\ amy.loutfi@oru.se, andrea.orlandini@istc.cnr.it, filippo.palumbo@isti.cnr.it, fpa@aass.oru.se, \\ stephen.vonrump@giraff.org, ales.stimec@xlab.si, jonas.ullberg@oru.se, britt.ostlund@ design.lth.se
}

\begin{abstract}
Early detection and adaptive support to changing individual needs related to ageing is an important challenge in today's society. In this paper we present a system called GiraffPlus that aims at addressing such a challenge and is developed in an on-going European project. The system consists of a network of home sensors that can be automatically configured to collect data for a range of monitoring services; a semi-autonomous telepresence robot; a sophisticated context recognition system that can give high-level and long term interpretations of the collected data and respond to certain events; and personalized services delivered through adaptive user interfaces for primary users. The system performs a range of services including data collection and analysis of long term trends in behaviors and physiological parameters (e.g. relating to sleep or daily activity); warnings, alarms and reminders; and social interaction through the telepresence robot. The latter is based on the Giraff telepresence robot, which is already in place in a number of homes. Particular emphasis is put on user evaluation outside the laboratories. A distinctive aspect of the project is that the GiraffPlus system will be installed and evaluated in at least $\mathbf{1 5}$ homes of elderly people. The concept of "useworthiness" is central in order to assure that the GiraffPlus system provides services that are easy to use and worth using. In addition, by using existing and affordable components we strive to achieve a system that is affordable and close to commercialization.
\end{abstract}

Keywords: Artificial Intelligence, Health Care and Assistive Devices, Human Machine Interaction, Smart Homes.

\section{INTRODUCTION}

$\mathrm{T}$ HE prolongation of independent living for promotion of a healthier society is a social and economic challenge. Most elderly people wish to remain in their homes as long as possible as this is in general conducive of a richer social life and paramount to maintaining established habits. To adhere to this wish is also positive from an economic perspective as the cost of care at home is almost always much less than the cost of residential care. However, several issues need to be addressed in order to prolong independent living. One is early detection of possible deterioration of health so that problems can be

The work has been supported via the EU FP7 programme via the GiraffPlus project. remediated in an early stage and timely involvement of health care providers and family can be assured. A second issue is to provide adaptive support which can offer services to assist in coping with age-related impairments. Third, ways of supporting preventive medicine must be found as it has been increasingly recognized that preventive medicine can contribute to promoting a healthy lifestyle and delay the onset of age-related illnesses.

The GiraffPlus system is developed in the GiraffPlus FP7 project and addresses the above challenges. The system consists of a network of home sensors that measure e.g. blood pressure or temperature, or detect e.g. whether somebody occupies a chair, falls down or moves inside a room. The data from these sensors are interpreted by an intelligent system in terms of activities, health and wellbeing: e.g. the person is exercising or the person is going to bed, or a fall has occurred. These activities can then trigger alarms or reminders to the primary user or his/her caregivers (secondary users), or be analyzed off line and over time by a health professional (secondary users). The system should automatically adapt to perform specific services such as checking the person's night activities. There is also a telepresence robot, the Giraff robot, which can be moved around in the home by somebody connected to it over Internet, e.g. a caregiver. The Giraff robot is effectively a mobile communication platform, equipped with video camera, display, microphone and speakers, and a touch screen, and it helps the user to maintain his/her social contacts. When a remote visitor uses the Giraff robot as a communication tool, what has happened in the home in terms of activities and the physiological measurements of the person can be seen and discussed. Both the remote visitor and the person in the home have access to the information and the system can be modified to assess other aspects with the agreement of the primary user.

Particular emphasis is put on user evaluation outside the laboratories. The GiraffPlus system will be installed and evaluated in at least 15 homes of elderly people distributed in three European countries (Italy, Spain and Sweden). These evaluations drive the development of the system. The concept of "useworthiness" is central in order to assure that the GiraffPlus system provides services that are easy and worth using. 


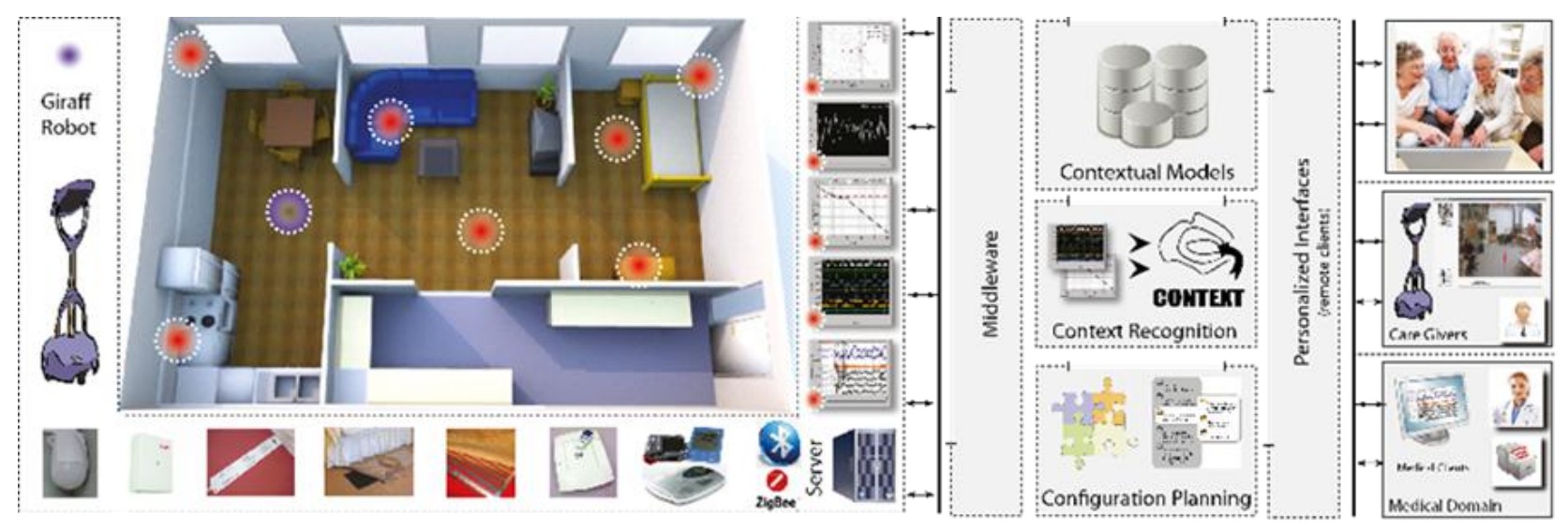

Fig. 1 The GiraffPlus structure

Fig 1 illustrates graphically the main components of the system. On the left the Giraff telepresence robot is shown. The robot uses a Skype-like interface to allow caregivers to virtually visit an elderly person in the home. The Giraff robot is enhanced with semi-autonomy in order to increase safety and ease-of-use. The GiraffPlus system also includes a network of sensors. Data from these sensors are processed by an advanced context recognition system based on constraint-based reasoning which both detects events on-line and can perform inference about long term behaviors and trends. Personalized interfaces for primary and secondary users are developed to access and analyse the information from the context recognition system for different purposes and over different time scales. An important feature of the system is an infrastructure for adding and removing new sensors seamlessly, and to automatically configure the system for different services given the available sensors. This is done using planning techniques. These features provide an adaptive support which facilitates timely involvement of caregivers and allows monitoring relevant parameters only when needed. In GiraffPlus, the business model development is to be woven with the evaluation activities and consequently the iterative approach also allows for user evaluation to be a major driver for the development of the business model and the exploitation strategy. Part of the user evaluation will be dedicated to investigate the potential for the services to be commercialized either through purchase, leasing or subscription models. From the onset of the project, all system architecture, functional specifications, feature development and other major technical decisions are evaluated against the emerging business model. In particular, feasibility of these issues with respect to practical cost, implementation and support considerations will be assessed. Interoperability, adherence to (or development of) relevant standards, and "open source" philosophy are all key factors in these assessments.

\section{NOVELTY OF THE GIRAFFPLUS SYSTEM}

The main novelty of the GiraffPlus system from the user perspective is the combination of: an easy communication tool, including the possibility to see the other person and to move in the environment; the possibility to have meaningful and personalized information about what has happened in the home; the collection and monitoring of physiological parameters; and the raising of alarms and warnings in case of need.
There is a clear need of a system with such functionalities in western societies where the elderly population is increasing and in fact there is already a market for products of this kind. A large number of companies have been newly established in most industrialized countries offering monitoring solutions where several parameters are monitored and information is sent via Internet and/or mobile phones. Most of the solutions use wireless motion or contact sensors on doorways, windows, walls, ceilings, cabinets, refrigerators, appliances or beds to track seniors' movements. The availability of many commercial solutions also shows a need for monitoring and an acceptance of the technology as a help.

The novelty of the GiraffPlus system with respect to such solutions is that it builds over the level reached by the latter and uses high level reasoning for context recognition, configuration planning and personalization and interaction services. No commercial system is currently available that addresses this aspect.

The novelty of the GiraffPlus system from a research perspective is the development of a system combining sensors and a teleoperated robot with high level reasoning including context recognition, configuration planning and personalization and interaction services. An important aspect that sets GiraffPlus apart from other research projects is the evaluation of the system in real homes for extended periods of time. This requires solutions that are reliable, robust and also acceptable from the users' perspectives. In addition the system needs to be perceived as really use-worthy to be used for such a length of time.

In recent years, the EU has funded a number of projects promoting independent living for elderly. The Ambient Assisted Living Joint Program (http://www.aal-europe.eu/) AAL, is focused exactly on this topic. Several AAL projects address smart homes for the elderly. The solutions proposed by the AAL projects are based on established technologies. They are developed in a close loop with the users, and the projects are expected to result in commercialization in the near future. However, the solutions tend to be specific for a certain class of illnesses and the scientific novelty can be limited. In many of them, services provided are mostly based on a one-to-one correspondence between sensor data and actions that the system performs. Conversely, GiraffPlus proposes to focus on changing needs via long term monitoring of complex behaviours and the use of high level reasoning. This allows the system to recognize and react to more complex human behaviours, and will support the provision of services in 
response to long-term physiological trends. These capabilities are paramount in early diagnosis and prevention, and constitute what we believe is a key factor for uptake.

The EU has also financed many FP6 and FP7 projects that have some similarities with the GiraffPlus project. The FP6 and FP7 projects tend to address more general problems with respect to the AAL projects and develop more advanced systems (often including autonomous robots). However, the results of these projects are farther from commercialization, both in terms of technological maturity and in terms of affordability. User evaluations tend to be more limited than those carried out in AAL projects. The GiraffPlus project combines a challenging research development with a thorough evaluation that is similar to the kind of evaluation done in an AAL project. Our effort on evaluating useworthiness is unique and from this point of view, we propose a middle ground between AAL-like user evaluations (which are appropriate for products that have a 2-year time to market) and Framework Programme projects (where user evaluation is focused purely on refining research prototypes). We also advocate a way to introduce the technology that is non-obtrusive and even enjoyable. Additional projects related to ours are the ones that aim at creating a common platform for AAL, like universAAL on top of which to develop intelligent software applications for the end users. It is our intention to be aligned to the universAAL results, to reuse the Open Source software released by them.

\section{USER REQUIREMENTS}

The GiraffPlus system is being developed on the basis of user requirements from both primary (elderly living in their apartment), and secondary (health care professional or family members and friends) users. Overall a total of $\mathbf{3 2 5}$ users (among primary and secondary) have been involved in this initial phase. Qualitative and quantitative research has been carried out in the three countries of Sweden, Spain and Italy, to elicit user requirements and expectations in terms of type of services as well as system design and appearance. The study has started with a literature review, followed by focus groups and questionnaires. Specifically, the results of the focus groups have been validated via questionnaires and workshop with users. Some qualitative cross-cultural analysis has also been performed in order to highlight differences emerged during the studies in the three countries. Result of this effort is a list of user requirements with an associated level of priority and a set of preferences on different mockups of a component of the system that can be both used to influence the future architecture definition and functional specification of the GiraffPlus system. This comprehensive list of requirements is not only of interest for the specific GiraffPlus system, but also outlines general preference of primary and secondary users with respect to technological solution.

\section{SYSTEM ARCHITECTURE}

To establish a clear correspondence between the user requirements and the implementation of the GiraffPlus system, the user requirements have been translated into technical requirements that are directing the architectural structure and the development of the system. In accordance with them an overall structure of the system has been outlined. Fig. 2 shows the general component diagram of the GiraffPlus system. In particular, four main components have been identified: Physical Environment and Software Infrastructure; Data Storage, Intelligent Monitoring and Adaptation Service; and finally Data Visualization, Personalization and Interaction Service.

The Physical Environment and Software Infrastructure component represents the basic level of functionalities of the GiraffPlus system. All the data services are grounded on functionalities of this part of the system. This module is also in charge of providing the common and interoperable communication service. In particular, the Middleware Infrastructure implements the Middleware service constituting a gateway shared among all the system components.

The role of the Sensors Network is to gather the data generated by the sensors (deployed in the elderly home) as well as to provide the rest of the system with the (possibly pre-processed) collected data.

The Giraff Robot component implements the GiraffPlus tele-presence functionalities, also providing a Pilot service to remotely control the robot.

The Data Storage component is responsible for providing a general database service for all the data generated by parts of the system and providing data access functionalities. Specifically, the role of this component is to manage a database containing all the data collected through the Middleware service and generated by other system components (for instance, the Sensors Network). Additionally, it enables other components to access the information and reason over it (also considering historical evolution).

The Intelligent Monitoring and Adaptation Service is the

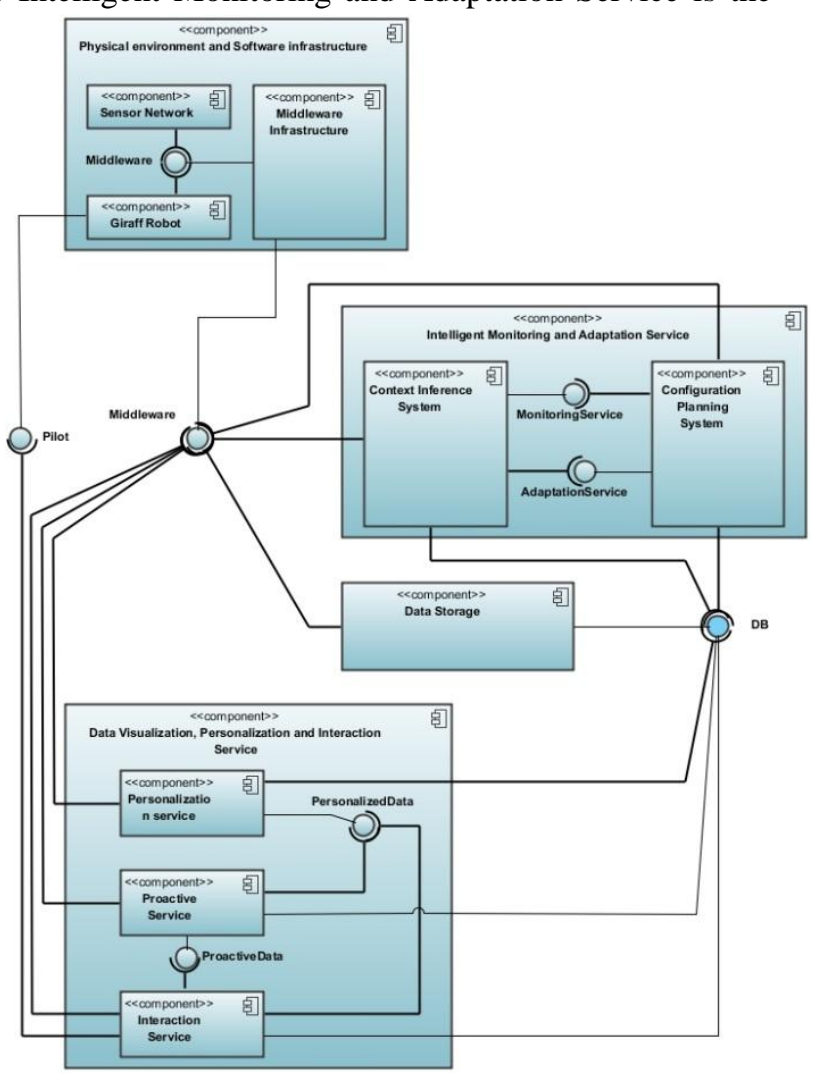

Fig. 2 the GiraffPlus system architecture 
component responsible for context/activity recognition and configuration planning. This part of the system encompasses two general reasoning systems namely:

- $\quad$ The Context Inference System, which is in charge of implementing the requested monitoring activities by means of context/activity recognition and relies on a timeline-based representation of the data generated by the sensors.

- The Configuration Planning System, which is responsible for providing suitable configuration settings for the Sensors Network according to the requested monitoring activities.

The Data Visualization, Personalization and Interaction Service is the part of the system responsible for creating user-oriented service. It provides different end-users with suitable interaction modalities for the available services and provides visualization services adapted to the different interaction created within the system. Depending on the classes of end-users, personalized services and specific dialogue boxes are offered which take into account his/her specific needs/roles.

The Personalization Service acts as a back-end of the Interaction service and is in charge of collecting and keeping up-to-date all the data needed to generate personalized interactions. It basically creates and dynamically maintains profiles for all the end users involved in GiraffPlus and also provides some reasoning services specifically tailored for the persons involved (e.g., the reminding setting and associated dialogues).

The Proactive Services are responsible for collecting specific functionalities to prepare content to be sent from the technological modules to users.

Finally, the interconnections among components are also relevant. In this regard, a crucial role is played by the Middleware service as it provides the central connection point that is shared by all the components according to the needed information exchanges.

\section{SENSORS}

The complete system uses a number of sensors that are pervasively integrated in the home or can be used by the elderly to collect vital signs measurements. The latter are provided by the IntelliCare Company. IntelliCare has developed and thoroughly tested a user-friendly system Look4MyHealth - that takes several vital sign measurements like weight, blood pressure, blood glucose through dedicated sensors, in a comfortable and thorough way at the patient's home. A portable pulse and oxymetry is also available. The environmental sensors are mostly provided by the Tunstall Company. These include smoke sensors, temperature sensors, fall sensors, sensors that provide door usage information (i.e. how many times the person uses a certain door in the apartment or the door of a cupboard), bed/chair occupancy information, and electric appliance usage. Fig. 6 shows some of the used physiological and environmental sensing devices.

It is especially important in this kind of application that the sensors are not perceived as compromising privacy and integrity of the elderly. For this reason we employ what we call a "ghost" approach. That is, the actual person is never seen, rather only the effects of his/her actions are detected by the sensors. For instance, data from door crossing and humidity sensors in the bathroom can be used to infer that somebody has taken a shower without ever seeing the person. In addition, integrity and security aspects are considered for the wireless communication when the personal data is transmitted.

\section{HIGH LEVEL REASONING}

Context recognition and the configuration planner are important components of the system in order to give meaning to the sensor data. The former recognizes the activities performed in the home (e.g. eating-dinner, sleeping, food-cooking, and robot-moving) on the basis of the sensors data. An activity has an extension in time, and it typically involves changes in observable state variables. The task of the configuration planner is to configure the sensor network in terms of subscriptions (and possibly also giving parameters to functionalities) so that the state variables requested by the context recognition system are monitored. Examples of configurations are shown in Fig. 3. It also directs this data to the context recognition system. For instance, the context recognition may ask the configuration planner to supply the state variable "person-in-room-A" and the latter can then generate and deploy a configuration. The configuration planner should also be able to adapt to changing conditions, e.g. functionalities added, removed, or malfunctioning, or changes in certain state variables (e. g. light $=$ off).
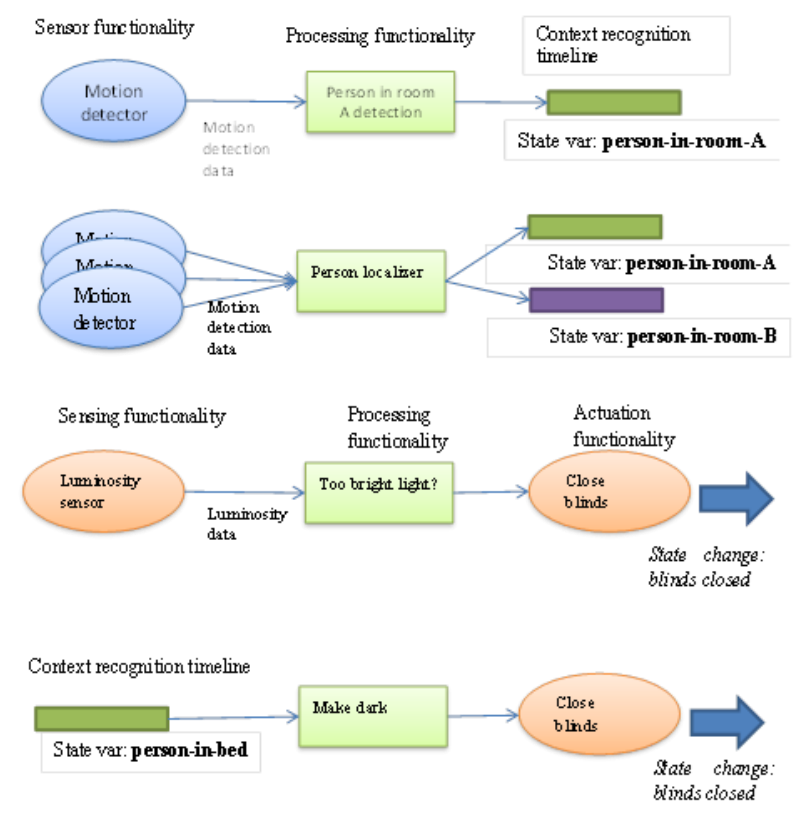

Fig. 3 Examples of configurations inferred from home sensorial data. 


\section{A. Configuration planning}

Given a task, the configuration planning is the problem of generating a functional configuration of a networked robot system consisting both of mobile robots and sensors and actuators distributed in the environment that solves that task. In a functional configuration, sensory, computational and motoric functionalities belonging to the different devices are connected with communication channels.

The work by Lundh et al. [1-3] offers a centralized solution to the problem, which has been successfully employed in an intelligent home test bed. A related approach for multi-robot systems is AsyM TRe-D [4, 5]. ASyMRTe-D works by connecting functionalities, belonging to different robots, into coalitions. The GiraffPlus configuration planner may produce configurations that change over time, in particular when actuation (pre- and postconditions of actions) is involved. The configuration planner makes the sensor network to provide the information (state variable values) that the context recognition system needs, and may also change state variables through actuation. A particular important capability of the GiraffPlus configuration planner is to handle many requests at the same time.

\section{B. Context recognition}

Context recognition is a fundamental capability of the proposed system. Context recognition addresses the general goal of providing the system with the capability of inferring context about the human user(s) and of the environment, including its ubiquitous sensors and actuators. In the particular sub-case of human context recognition, this capability often goes under the name of activity recognition.

Current approaches to the problem of recognizing human activities can be categorized as data-driven or modeldriven. In data-driven approaches, models of human behaviour are acquired from large volumes of data over time. Notable examples of this approach employ Hidden Markov Models (HMMs) in conjunction with learning techniques for inferring transition probabilities [6, 7]. Model-driven approaches follow a complementary strategy in which patterns of observations are modeled from first principles rather than learned or inferred from large quantities of data $[8,9]$.

Data- and model-driven approaches have complementary strengths: the former provide an effective way to recognize elementary activities from large amounts of continuous data; conversely, model-driven approaches are useful when the criteria for recognizing human activities are given by crisp rules that are clearly identifiable. More importantly, the same inference mechanism that is used for model-based activity recognition can be leveraged for plan synthesis. This is particularly so in cases where the expressiveness of the modeling language affords plan-generation and execution monitoring capabilities, such as the SAM framework that is used in this system [10-12].

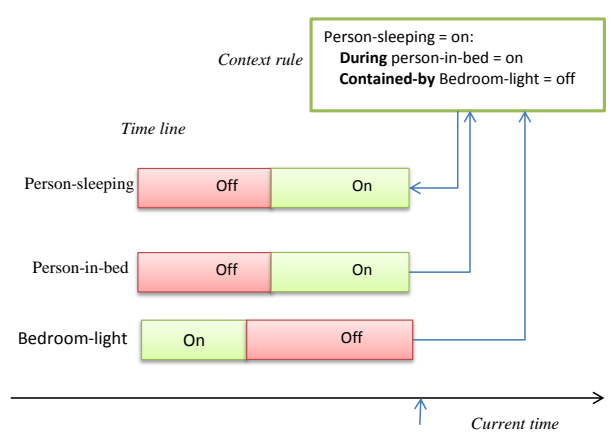

Fig. 4 Example of time line (simplified) where an activity (Person-sleeping) is inferred from state variables obtained from sensor network (Person-in-bed and Bedroom-light) according to a context rule.

The context recognition system deals with activities. An activity has an extension in time, and it typically involves changes in state variables (e.g. eating-dinner, sleeping, food-cooking, and robot-moving). Similar constraint-based approaches to inference in the domain of domestic activity monitoring include [13, 14].

Given the requests from services, the context recognition system determines what state variables need to be monitored and requests these variables from the configuration planning system. It then continuously receives data about these state variables from the sensor network, enters them into the time line and derives activities from them, see Fig. 5. The context recognition system may also change its request for state variables to the configuration planner, depending on what activities comes up or are expected to come up. And it may request changes of state variables (actuation) when certain activities are triggered.

Some physiological state variables, e.g. ECG, are not suitable for processing by the context recognition system but should just be stored for later processing with more adequate tools. However, it is important to maintain the relation (e.g. temporally) to the information in the time line (database).

\section{VisualiZATiON}

A preliminary visualization module has been realized that presents an integrated interface showing both the activities and physiological parameters and the pilot component used to steer the Giraff robot. An example can be seen in Fig. 5. This visualization has been assessed in an evaluation session with secondary users and the feedback is now used in the development of a new prototype. 

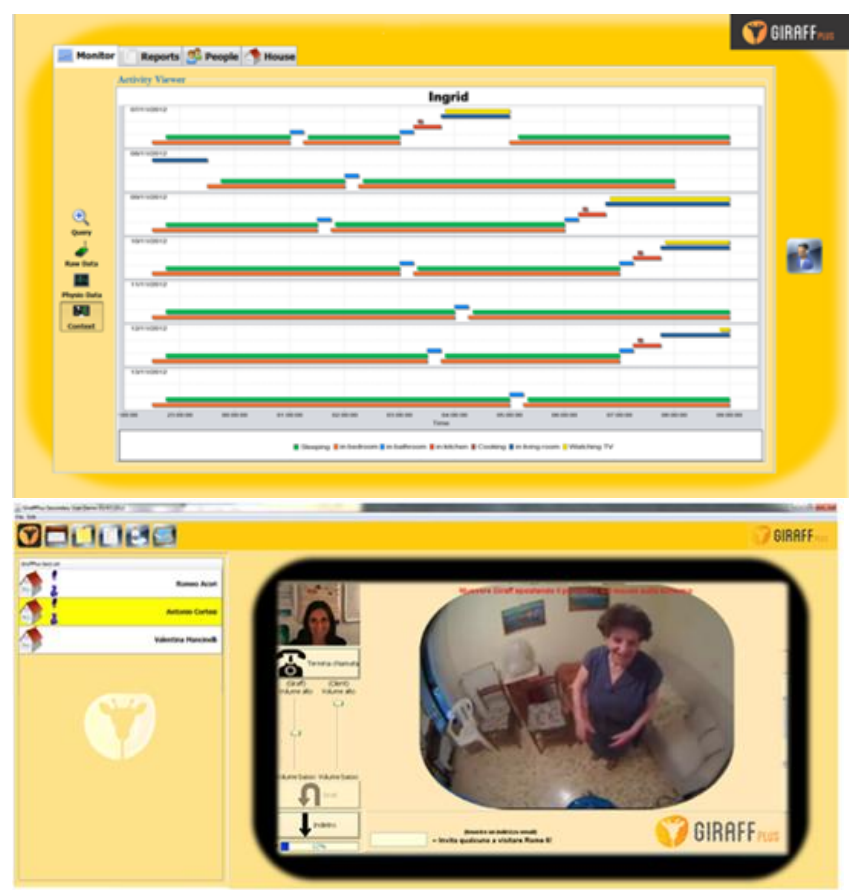

Fig. 5 Upper graph, visualization of night activities: green line - sleeping; orange line - in bedroom; light blue line - in bathroom; light red line - in kitchen; dark red line cooking; dark blue line - In living room; yellow line Watching TV. Lower display, interface for the remote driver of the Giraff robot

A visualization for the primary users is also under development. Here the challenge is mainly how the data should be presented in the best way given the heterogeneity of the primary users in terms of ease of use of technology.

\section{TESTING OF THE SYSTEM}

A first integrated version of the system has been implemented in the pilot apartment Ängen in Örebro (Fig. 6). The test apartment Ängen is located in a unique building complex as part of an initiative to provide complete care facilitates for older people in Örebro, both elderly and independent seniors.

The aim has been to create a preliminary version of the system including the Giraff robot, the IntelliCare sensors providing physiological data, a physiological sensor for pulse oximetry measurements, the monitoring sensors from Tunstall and middleware. This version of the system has been used for providing data to the context recognition and configuration planning components and in a preliminary evaluation of the system with primary and secondary users. The system has been tested in two realistic scenarios considered relevant by the secondary users and recorded in a video. In the first scenarios an elderly person with diabetes receives regular visits from a nurse and discusses with her the physiological data collected by the GiraffPlus system. The system is adapted to the need of the person first by adding the monitoring of night activities for assessing sleep, then by adding the monitoring of falls. In both cases the changes of the system are discussed and agreed between the primary and secondary user. The result of the night activities monitoring is shown in Fig. 5. In the second scenario a physiotherapist gives advice and checks the
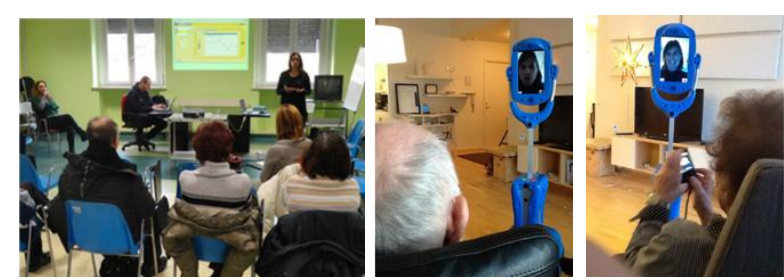

Fig. 7 Evaluation of the GiraffPlus system with secondary users and with primary users.

exercise routine and the movement done during the day of a primary user.

\section{EVALUATION WITH USERS}

The evaluation is central in GiraffPlus. Gerontechnologyrelated research and several design efforts have contributed to including older users of technology in the evaluation and widening the scope from ageing as merely a decline to ageing as a life phase with possibilities despite health problems [15, 16]. Wellness, barrier-free societies and "universal design"/"design for all", are concepts that take elderly seriously by pursuing communication solutions that include the elderly in e-society and help create mainstream products rather than tools that stigmatize old age. In this regard, design can act both as a conservative and a liberating force. In the development of the GiraffPlus system an active role is given to the elderly as part of the design process. Often there is confusion about the way that we would prefer elderly to behave with how they actually behave. The novelty of this project lies in the implementation of a design process where knowledge of technology's relationship in everyday life is used to understand how GiraffPlus may be use-worthy and what facilitates and prevents its use. This approach moves forward the development of technical solutions that support aging in modern society in four ways:

By strengthening elderly's influence in the design process to optimize the output: This project deliberately avoids prejudices of old age by involving elderly who wish to live a continued independent life in modern society. The elderly are often considered as a homogeneous group ascribed particular difficulties to absorb and use new technologies. One of the signs of the willingness to change this view is the initiative among elderly themselves to contribute to good practice [17]. This project involves elderly as active and mature users of technology that are experts on their own needs and demands. Old users can often be described with the sociological concepts of being "lay end users" or "implicated users" [18]. The concept of "lay end users" was introduced to highlight some end user's relative exclusion from expert discourse. Implicated actors are those silent or not present but affected by the action. In this project the elderly are involved as active subjects throughout the design process and take part in the discourse throughout the iterative design process.

By departing from a traditional technocentric model to develop more person-centered approaches [19, 20] and to explore experiences of technology as sources for innovations [21]: In this project, we allow older users to express their problems and life-long habits as users of technology. They are given the opportunity to express their obvious and visible needs as well as the latent needs that can be awakened in the context of playfulness as a tool in 
the design process [22]. People who invest something of themselves in a product are increasing their emotional ties (attachment) to the product [23, 24]. In this project the elderly have been given the opportunity to participate in a creative way in the development of three mock-ups of the GiraffPlus and to develop their own relationship to this application [25]. Today digital devices have become an extension of us, not as a gadget that symbolizes a lifestyle but as an actual part of our conscious self [26]. Uniquely in this project elderly are given the opportunity to shape a technology - the GiraffPlus system - in a creative and playful way.

By testing technology and services in a real life environment and evaluate them also considering how the system fits into the users' everyday life. Important concepts in this respect are "useworthiness" and "usability". While usability is a test on whether an application is functional and useful, useworthiness is a test of whether the application is worth using; i.e. can fulfill the needs that are the most important ones to the person [27, 28]. Both usability and useworthiness have a bearing on human experience and how the encounter with new technologies affects them. In particular it affects their opinion of whether the new application is worth a try, if it has comparative advantages with respect to other solutions and their opinion of whether the technology will affect their own identity and status in relation to others. The feeling of being involved in an innovation process that is placed at the front end of technology development is the kind of feeling that we want to inspire in the users of GiraffPlus. Useworthiness, unlike usability, has a clear bearing on the context. When new technologies are introduced in the home a domestication process starts in which technology is ascribed practical and symbolic meanings that lead to its becoming integrated or rejected $[29,30]$. Users make the technology "their own" when it is integrated in practice and in social relationships. By contributing to the field of technology acceptance modeling, through the application of state-of-the art technology acceptance models. User acceptance has been defined as "the demonstrable willingness within a user group to employ technology for the tasks it is designed to support" [31]. A number of theoretical models have been widely used in the literature for modeling user acceptance of technology. TAM [32] is one of the most widely used models. It suggests that Perceived Usefulness and
Perceived Ease of Use influence the Behavioral Intention to use a system through the mediation of Attitude. A more recent and comprehensive model is the Unified Theory of Acceptance and Use of Technology (UTAUT) [33]. It is also important to study the psychosocial impact of a technological aid on its users in order to identify the reasons for its use or its abandonment and recognize problematic areas. Psychosocial factors may be crucial in determining the degree of satisfaction of the user; sometimes the abandonment of an assistive device is due to technical problems but there are several cases where factors such as personality and motivation can have an important effect. Satisfaction and frequency of use are determined by real costs and benefits related to use of assistive device [34].

A first evaluation has been performed in the Ängen apartment with primary users to assess a preliminary version of the system and its perceived use-worthiness and with secondary users with respect to the visualization of the data. The next step is to test the intermediary GiraffPlus prototype in the homes of elderly users in Italy, Spain and Sweden. In preparation to this important evaluation ethical guidance has been jointly developed between the partner countries where evaluations are being performed; in addition criteria and recruitment plans have been established and a method for user involvement has been developed. The results from the preliminary GiraffPlus prototype evaluation with primary and secondary users (Fig. 7) show the need for contextualization and customization of scenarios and technical possibilities. It proves the importance of integrating services into the everyday life of elderly and into the organization of health care systems and daily work among health care professionals.

\section{CONCLUSIONS}

In order to sustain a good quality of life for an aging population, independent living is a key requirement. Three elements must be in place: 1) the elderly must be of sufficient health to live safely at home; 2) there must be the opportunity for good social interaction habits within the home to avoid isolation and loneliness; 3) intervention must occur in a timely fashion that is beneficial to the elderly and efficient for the caregiver system. Creating an infrastructure to continuously monitor activities of persons
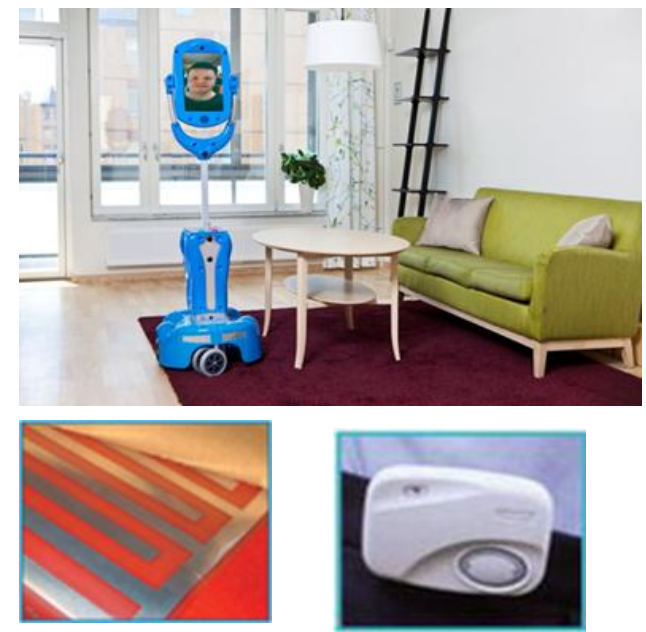

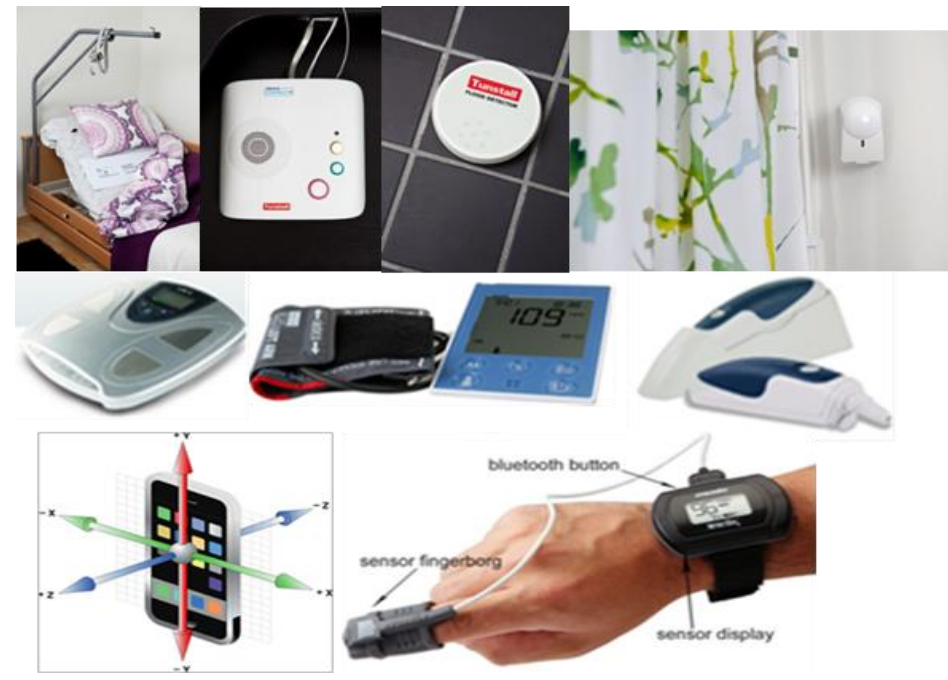

Fig. 6 The Giraff robot in the test apartment. Examples of Tunstall sensors: first line from left to right: Bed/Chair Occupancy Sensor - Gateway collecting sensor data - Flood detector - Fast PIR (Movement Detector); second line from right Enuresis Sensor - Fall Detector - Fast PIR (Movement Detector). Sensors from IntelliCare: in the middle from left to right: scale, blood and glycose sensor, thermometer. Portable pulse and oxygen sensor. 
in the home enables an assessment of health degradation in a manner which has not been previously possible. Such an assessment aims to contribute to a reduction in healthcare costs as many health ailments can be addressed in an early stage of on-set. For the elderly, the concept pursued in GiraffPlus provides a tool to address concerns about isolation and safety by encouraging social contact and thereby increasing quality of life and possibilities to prolong independent living. For the caregiver, the specific technologies to be developed allow for continuous information about the status of the elderly, encourage a meaningful interaction concerning patient wellbeing, enable timely involvement, provide time effective care and not least lead to economical savings.

The integration and deployment effort put forth by this project constitutes a significant step towards applying artificial intelligence in a real-world context where usual simplifying assumptions cannot be made. Thus, in addition to providing solutions for an extremely important future market niche for European industry, we expect that GiraffPlus system will have a strong impact with respect to the advancement of European excellence in smart environment development.

\section{REFERENCES}

[1] Lundh R, Karlsson L, Saffiotti A (2008) Autonomous Functional Configuration of a Network Robot System. Robotics and Autonomous Systems 56(10):819-830

[2] Lundh R, Karlsson L, Saffiotti A (2008) Automatic Configuration of Multi-Robot Systems: Planning for Multiple Steps. Proc. of the 18th European Conference on Artificial Intelligence (ECAI). Patras, Greece

[3] Lundh R (2009) Robots that Help Each-Other: Self-Configuration of Distributed Robot Systems. PhD Thesis. Örebro University, Sweden

[4] Tang F, Parker LE (2005) Distributed multi-robot coalitions through ASyMTRe-D. Proceedings of the IEEE/RSJ International Conference on Intelligent Robots and Systems (IROS), Alberta

[5] Parker LE, Tang F (2006) Building multi-robot coalitions through automated task solution synthesis. Proceedings of the IEEE, special issue on Multi-Robot Systems, 94 (7) pp 1289-1305

[6] Patterson D, Fox D, Kautz H, Philipose M (2005) Fine-grained activity recognition by aggregating abstract object usage. Proc. of the 9th IEEE International Symposium on Wearable Computers

[7] Wu J, Osuntogun A, Choudhury T et al (2007) A Scalable Approach to Activity Recognition Based on Object Use. Proceedings of ICCV 2007. Rio de Janeiro, Brazil

[8] Goultiaeva A, Lespérance Y. (2007) Incremental plan recognition in an e agent programming framework. Working Notes of the AAAI Workshop on Plan, Activity, and Intention Recognition (PAIR)

[9] Jakkula V, Cook D, Crandall A. (2007) Temporal pattern discovery for anomaly detection in a smart home. Proc. of the 3rd IET Conf. on Intelligent Environments (IE), pp. 339-345

[10] Cirillo M, Pecora F, Saffiotti A (2010) Proactive assistance in peis-ecologies: a constraint-based approach. Handbook of Research on Ambient Intelligence: Trends and Perspectives IGI Global

[11] Pecora F, Cirillo M (2009) A Constraint-Based Approach for Plan Management in Intelligent Environments. Proc. of the Scheduling and Planning Applications Workshop at ICAPSO9

[12] Ullberg J, Loutfi A, Pecora F (2009) Towards Continuous Activity Monitoring with Temporal Constraints. Proc. of the 4th Workshop on Planning and Plan Execution for Real-World Systems at ICAPS09

[13] Pollack M, Brown L, Colbry D et al (2003) Autominder: an intelligent cognitive orthotic system for people with memory impairment. Robotics and Autonomous Systems (44) pp. 273-282

[14] Cesta A, Cortellessa G, Rasconi R et al (2010) Monitoring elderly people with the robocare domestic environment: Interaction synthesis and user evaluation. Computational Intelligence, Special Issue on Scheduling and Planning Applications

[15] Graafmans J, Taipale V, Charness N. (eds) (1998) Gerontechnology. A Sustainable Invetsment in the Future. Amsterdam: IOS-Press
[16] Sixsmith A, Gutman G (2010) Proceedings of the 7th Gerontechnology Conference

[17] The Senior project (2009) Good Practices in e-Inclusion, ethical guidance and designing a dialogue roadmap. A senior project within the seventh framework programme. Deliverable D4.1

[18] Oudshoorn N, Pinch T (eds) (2005) How Users Matter. The Co-construction of Users and Technology MIT Press

[19] Sinden D, Wister A (2008) E-health promotion for aging baby boomers in North America. Gerontechnology 7 (3) pp.271-278.

[20] Dickinson A, Dewsbury G (2006) Designing computer technologies with older people. Gerontechnology (5) 1 pp 1-3

[21] Kohlbacher F, Herstatt C (2011) The Silver market Phenomen. Business Opportunities in an Era of Demograhic Change. Berlin: Springer-Verlag

[22] Danhauer SC, Carlson CR, Andrykowski MA (2005) Positive psychosocial functioning in later life: use of meaning-based coping strategies by nursing home residents. Journal of Applied Gerontology (24) pp. 299-318.

[23] Mugge R. (2007) Product attachment. Doctoral thesis, Delft University of Technology, Faculty of industrial design engineering, Department of product innovation and management

[24] Schifferstein H., Zwartkruis-Pelgrim E (2008) Consumer-product attachment: Measurement and Design Implications. International Journal of Design, 2(3) pp.1-13.

[25] Markussen T (2009) Bloody Roots as Emotional design: How Emotional Structures May Change Expectations of technology Use in Hospitals. International Journal of Design 3 (2) pp.27-39.

[26] Turkle Sh (ed) (2007) Evocative objects. Things we think with Cambridge, Mass: MIT Press

[27] Rubin J, Chisnell D (2008) Handbook of Usability Testing, Second Edition. Wiley

[28] Eftring H (1999) The Useworthiness of Robots for People with Physical Disabilities. Doctoral Dissertation 1:1999. Lund University. Department of Design Sciences, Certec.

[29] Sorensen K (2005) Domestication: the enactment of technology. Domestication of Media and Technology. Maindenhead: Open University Press, pp. 40-62

[30] Silverstone R (2005) Media, technology and everyday life in Europé. Aldershot: Ashgate

[31] Dillon A (2001) Encyclopedia of Human Factors and Ergonomic Ed. London: Taylor and Francis, ch. User Acceptance of Information Technology.

[32] Davis F (1986) A Technology Acceptance Model for Empirically Testing New End-User Information Systems: Theory and Results. Ph.D. dissertation, M.I.T. Boston (MA)

[33] Venkatesh V, Morris M G, Davis G B, Davis F (2003) User Acceptance of Information Technology: Toward a Unified View. MIS Quarterly, vol. 27, no. 3, pp. 425-478

[34] Vash C (1983) Psychological Aspects of Rehabilitation Engineering," in Technology for Independent Living. Proceedings from the 1981 Workshops on Science and Technology for Handicapped. AAAS 